TRANSACTIONS OF THE

AMERICAN MATHEMATICAL SOCIETY

Volume 365, Number 9, September 2013, Pages 4881-4894

S 0002-9947(2013)05777-7

Article electronically published on March 19, 2013

\title{
PROOF OF A CONJECTURE BY AHLGREN AND ONO ON THE NON-EXISTENCE OF CERTAIN PARTITION CONGRUENCES
}

\author{
CRISTIAN-SILVIU RADU
}

Abstract. Let $p(n)$ denote the number of partitions of $n$. Let $A, B \in \mathbb{N}$ with $A>B$ and $\ell \geq 5$ a prime, such that

$$
p(A n+B) \equiv 0 \quad(\bmod \ell), \quad n \in \mathbb{N} .
$$

Then we will prove that $\ell \mid A$ and $\left(\frac{24 B-1}{\ell}\right) \neq\left(\frac{-1}{\ell}\right)$. This settles an open problem by Scott Ahlgren and Ken Ono. Our proof is based on results by Deligne and Rapoport.

\section{INTRODUCTION}

Let $p(n)$ denote the number of partitions of the positive integer $n$. Ramanujan 25] proved the following congruences,

$$
\begin{aligned}
& p(5 n+4) \equiv 0 \quad(\bmod 5), \\
& p(7 n+5) \equiv 0 \quad(\bmod 7),
\end{aligned}
$$

and

$$
p(11 n+6) \equiv 0 \quad(\bmod 11) .
$$

We define $p(0):=1$ and $p(n):=0$ if $n<0$.

Following [4, let $\delta_{\ell}$ be defined by

$$
\delta_{\ell}:=\frac{\ell^{2}-1}{24} .
$$

Then the Ramanujan congruences above may be written in the form

$$
p\left(\ell n-\delta_{\ell}\right) \equiv 0 \quad(\bmod \ell), \quad n \in \mathbb{Z} .
$$

Ahlgren and Boylan 22 proved that (1) holds only for $\ell=5,7,11$. This question was asked by Ramanujan in [25] and is one of the few results on non-existence of congruences for the partition function within infinitely many arithmetic progressions. The proof is partially based on a result by Kiming and Olsson [15 which, to the best of our knowledge, is the first paper that proves non-existence of congruences within infinitely many arithmetic progressions. In both papers [2, 15] the tools involved are modular forms modulo $p$ as developed by Serre and SwinnertonDyer [28, 29]. This paper provides another non-existence result, but our methods are entirely different from the ones used in [2, 15].

Received by the editors February 24, 2011 and, in revised form, June 16, 2011 and December 7, 2011.

2010 Mathematics Subject Classification. Primary 11P83.

Key words and phrases. Partition congruences of Ramanujan type, Ahlgren, Ono conjecture. The author was supported by DK grant W1214-DK6 of the Austrian Science Funds FWF.

(c) 2013 American Mathematical Society 4881 
Many papers have been written on these three congruences and their extensions (already conjectured, and in some cases proved, by Ramanujan) to arbitrary powers of 5,7, and 11; see the fundamental works of Andrews, Atkin, Berndt, Dyson, Garvan, Kim, Ono, Ramanujan, Stanton and Swinnerton-Dyer [5, 6, 7, 9, 11, 13, 14, 26, 23, 24, 25. According to [3] each of these extensions lies within the class $-\delta_{\ell}(\bmod \ell)$. We say that a congruence

$$
p(A n+B) \equiv 0 \quad(\bmod \ell), \quad n \in \mathbb{Z}
$$

lies within the class $\beta(\bmod \ell)$ iff $\{A n+B\} \subseteq\{\ell n+\beta\}$. Here for integers $x, y$ we define $\{x n+y\}:=\{x n+y: n \in \mathbb{Z}\}$.

The important role that the class $-\delta_{\ell}(\bmod \ell)$ plays in the theory is illustrated by the work of Kiming and Olsson [15, who proved that if $\ell \geq 5$ is prime and $p(\ell n+\beta) \equiv 0(\bmod \ell)$ for all $n$, then $\beta \equiv-\delta_{\ell}(\bmod \ell)$.

Atkin, Newman, O'Brien, and Swinnerton-Dyer [7, 8, 10, 19] found further congruences modulo $\ell^{m}$ for primes $\ell \leq 31$ and small $m$. Examples by Atkin and Newman in [7] and [19] show that not every congruence lies within the class $-\delta_{\ell}$ $(\bmod \ell)$. For example when considering $\ell=13$, we have

$$
p(17303 n+237) \equiv 0 \quad(\bmod 13) .
$$

Ahlgren and Ono [1, 20] proved that if $\ell \geq 5$ is prime and $m$ is any positive integer, then there are infinitely many congruences of the form

$$
p(A n+B) \equiv 0 \quad\left(\bmod \ell^{m}\right) .
$$

As in the case of Ramanujan's congruences, all of these congruences lie within the class $-\delta_{\ell}(\bmod \ell)$. According to [3]: "the research described up to now consists of a systematic theory of congruences that lies within the class $-\delta_{\ell}(\bmod \ell)$, as well as some sporadic examples (as (2)) of congruences that fall outside of this class. In view of this, it is natural to wonder what role the class $-\delta_{\ell}(\bmod \ell)$ truly plays". In their paper [3], Ahlgren and Ono show that in general this class is not as distinguished as one might have expected. Namely, they prove that this class is one of $(\ell+1) / 2$ classes modulo $\ell$ in which the partition function enjoys similar congruence properties. The authors naturally ask if, for the remaining $(\ell-1) / 2$ classes, there are congruences or not. In [3, Sect. 1] the authors write: "In Section 4 , we consider those progressions $\ell n+\beta$ for $\beta \notin S_{\ell}$. We give heuristics that cast doubt on the existence of congruences within these progressions". In particular, Theorem 1.2 the main result of this paper, implies that indeed there are no such congruences. Before stating Theorem 1.2, we define for $\ell \geq 5$ a prime,

$$
\epsilon_{\ell}:=\left(\frac{-6}{\ell}\right)
$$

and

$$
S_{\ell}:=\left\{\beta \in\{0, \ldots, \ell-1\}:\left(\frac{\beta+\delta_{\ell}}{\ell}\right)=0 \text { or }-\epsilon_{\ell}\right\} .
$$

Remark 1.1. For $\ell \geq 5$ a prime, we have

$$
S_{\ell}=\left\{\beta \in\{0, \ldots, \ell-1\}:\left(\frac{24 \beta-1}{\ell}\right) \neq\left(\frac{-1}{\ell}\right)\right\} .
$$

Motivated by the results in [3, our main result Theorem 1.2 was conjectured by Scott Ahlgren and Ken Ono in another joint paper [4, Conj. 5.4]. 
Theorem 1.2. Suppose that $\ell \geq 5$ is prime, $A, B \in \mathbb{N}$ such that $A>B$ and

$$
p(A n+B) \equiv 0 \quad(\bmod \ell), \quad n \in \mathbb{N} .
$$

Then $\ell \mid A$ and there exists $\beta \in S_{\ell}$ such that $B \equiv \beta(\bmod \ell)$.

The proof of Theorem 1.2 is based on results by Deligne and Rapoport 12 and some results in 18 or $[22$. We are also using Theorems $4.2,4.3$ and 4.4 which were proven in a previous paper [21] and are also based on results in [12] and [18, or [22]. Our main contribution to the proof of Theorem 1.2 is Lemma 5.1] which takes the main part of the last section.

The organization of this paper is as follows. In Section 2 we prove Theorem 1.2 by citing several theorems in Section 4 . In Section 3 we give some preliminaries to modular forms. In Section 4 we make a classification of congruences in the sense that we show that some congruences $p(A n+B) \equiv 0(\bmod \ell)$ imply that $p\left(A^{\prime} n+B^{\prime}\right) \equiv 0 \bmod \ell$, where $\{A n+B\} \subseteq\left\{A^{\prime} n+B^{\prime}\right\}$. In Section 5 we prove our main contribution Lemma 5.1 which is needed for proving Lemma 4.7 in Section 4.

\section{THE PROOF}

Let $A, B \in \mathbb{N}$ with $A>B$ such that

$$
p(A n+B) \equiv 0 \quad(\bmod \ell), \quad n \in \mathbb{N} .
$$

Then by Theorem 4.3 there exists a positive integer $Q$ coprime to 6 dividing $A$ and a $\bar{t} \in\{0, \ldots, Q-1\}$ with $\bar{t} \equiv B(\bmod Q)$ such that

$$
p(Q n+\bar{t}) \equiv 0 \quad(\bmod \ell), \quad n \in \mathbb{N} .
$$

Then $\ell \mid Q$ because if not, Theorem 4.4 implies that there exists a positive integer $n_{0}$ such that $\ell \nmid p\left(Q n_{0}+\bar{t}\right)$ which contradicts (3). Hence we may write $Q=Q_{0} \ell^{r}$ for some positive integers $Q_{0}, r$ with $\operatorname{gcd}\left(Q_{0}, \ell\right)=1$. Now if $24 \bar{t}-1 \equiv 0(\bmod \ell)$, then we are finished. So assume that $24 \bar{t}-1 \not \equiv 0(\bmod \ell)$. Then by Lemma 4.5,

$$
p\left(Q_{0} \ell n+\bar{t}^{*}\right) \equiv 0 \quad(\bmod \ell), \quad n \in \mathbb{N},
$$

where $\bar{t}^{*}$ is the minimal non-negative integer such that $\bar{t}^{*} \equiv \bar{t}\left(\bmod Q_{0} \ell\right)$. Next we apply Lemma 4.7 to the congruence (44) and we obtain $\left(\frac{24 \bar{t}^{*}-1}{\ell}\right) \neq\left(\frac{-1}{\ell}\right)$ which together with Remark 1.1 above implies Theorem 1.2 .

\section{Preliminaries}

For $f$ an analytic function on the complex upper half plane $\mathbb{H}$ and $\gamma=\left(\begin{array}{ll}a & b \\ c & d\end{array}\right) \in$ $\mathrm{SL}_{2}(\mathbb{Z})$ (the set of all $2 \times 2$ matrices with integer entries and determinant 1 ), we define

$$
\left(\left.f\right|_{k} \gamma\right)(\tau):=(c \tau+d)^{-k} f(\gamma \tau), \quad \tau \in \mathbb{H}
$$

where

$$
\gamma \tau:=\frac{a \tau+b}{c \tau+d}
$$

For every positive integer $M$, we denote by $\Gamma(M)$ the set of all matrices in $\mathrm{SL}_{2}(\mathbb{Z})$ congruent to $\left(\begin{array}{ll}1 & 0 \\ 0 & 1\end{array}\right)$ modulo $M$. For $k$ an integer and $\Gamma$ a subgroup of $\mathrm{SL}_{2}(\mathbb{Z})$ containing $\Gamma(N)$ for some minimal $N$, we denote by $M_{k}(\Gamma)$ the set of all analytic functions on $\mathbb{H}$ such that:

- for all $\gamma \in \Gamma$ we have $\left.f\right|_{k} \gamma=f$; 
- for all $\xi \in \mathrm{SL}_{2}(\mathbb{Z})$ the function $\left(\left.f\right|_{k} \xi\right)(\tau)$ admits a Laurent series expansion (with finite principal part) in the variable $q_{N}:=e^{2 \pi i \tau / N}$. We call this expansion the $q$-expansion of $\left.f\right|_{k} \gamma$.

For $N$ a positive integer, let

$$
\Gamma_{0}(N):=\left\{\left(\begin{array}{ll}
a & b \\
c & d
\end{array}\right) \in \mathrm{SL}_{2}(\mathbb{Z}): c \equiv 0 \quad(\bmod N)\right\}
$$

and

$$
\Gamma_{1}(N):=\left\{\left(\begin{array}{ll}
a & b \\
c & d
\end{array}\right) \in \mathrm{SL}_{2}(\mathbb{Z}): a, d \equiv 1 \quad(\bmod N), c \equiv 0 \quad(\bmod N)\right\} .
$$

In particular $\Gamma(N) \subseteq \Gamma_{1}(N) \subseteq \Gamma_{0}(N)$. For our purposes, we will need the set

$$
\Gamma_{0}(N)^{*}:=\left\{\left(\begin{array}{ll}
a & b \\
c & d
\end{array}\right) \in \Gamma_{0}(N): a, c>0, \operatorname{gcd}(a, 6)=1\right\}
$$

and the group

$$
\Gamma_{1}^{-}(N):=\left\{\left(\begin{array}{ll}
a & b \\
c & d
\end{array}\right) \in \Gamma_{0}(N): a^{2} \equiv 1 \quad(\bmod N)\right\} .
$$

Note in particular that $\Gamma_{1}^{-}(N) \supseteq \Gamma_{1}(N)$.

Lemma 3.1. $\Gamma_{1}^{-}(N)$ is generated by $\Gamma_{0}(N)^{*} \cap \Gamma_{1}^{-}(N)$.

Proof. First note that

$$
\underbrace{\left(\begin{array}{cc}
6 N-1 & -1 \\
6 N & -1
\end{array}\right)}_{\in \Gamma_{0}(N)^{*} \cap \Gamma_{1}^{-}(N)} \underbrace{\left(\begin{array}{cc}
1 & -(h+1) \\
6 N & 1-6 N(h+1)
\end{array}\right)}_{\in \Gamma_{0}(N)^{*} \cap \Gamma_{1}^{-}(N)}=\left(\begin{array}{cc}
-1 & h \\
0 & -1
\end{array}\right)
$$

and

$$
\left(\begin{array}{cc}
-1 & 0 \\
0 & -1
\end{array}\right)\left(\begin{array}{cc}
-1 & h \\
0 & -1
\end{array}\right)=\left(\begin{array}{ll}
1 & h \\
0 & 1
\end{array}\right)
$$

Note that the two terms on the left hand side of (6) may be written as a product of matrices in $\Gamma_{0}(N)^{*} \cap \Gamma_{1}^{-}(N)$. Finally let $\left(\begin{array}{ll}a & b \\ c & d\end{array}\right) \in \Gamma_{1}^{-}(N)$ with $c>0$. Then,

$$
\left(\begin{array}{ll}
a & b \\
c & d
\end{array}\right)=\left(\begin{array}{cc}
\operatorname{sgn}(c) & t \operatorname{sgn}(c) \\
0 & \operatorname{sgn}(c)
\end{array}\right) \underbrace{\left(\begin{array}{cc}
a \operatorname{sgn}(c)-|c| t & b \operatorname{sgn}(c)-d t \operatorname{sgn}(c) \\
|c| & d \operatorname{sgn}(c)
\end{array}\right)}_{\in \Gamma_{0}(N)^{*} \cap \Gamma_{1}^{-}(N)},
$$

where $t \in \mathbb{Z}$ is chosen such that $a \operatorname{sgn}(c)-|c| t>0$ and $\operatorname{gcd}(a \cdot \operatorname{sgn}(c)-|c| t, 6)=1$. Since we already showed in (5) and (6) that $\left(\begin{array}{cc}\operatorname{sgn}(c) & t \operatorname{sgn}(c) \\ 0 & \operatorname{sgn}(c)\end{array}\right)$ is a product of matrices in $\Gamma_{0}(N)^{*} \cap \Gamma_{1}^{-}(N)$, the proof is finished.

\section{A ClassificAtion OF CONGRUENCES}

Definition 4.1. For $m$ a positive integer and $t \in\{0, \ldots, m-1\}$, we define $P_{m}(t)$ to be the set of all $t^{\prime} \in\{0, \ldots, m-1\}$ such that

$$
t^{\prime} \equiv t a^{2}+\frac{1-a^{2}}{24} \quad(\bmod m),
$$

for some $a \in \mathbb{Z}$ with $\operatorname{gcd}(a, 6 m)=1$. 
The following three theorems were proven in [21].

Theorem 4.2. Let $l, m$ be positive integers and $t \in\{0, \ldots, m-1\}$ such that

$$
p(m n+t) \equiv 0 \quad(\bmod l), \quad n \in \mathbb{N} .
$$

Then for all $t^{\prime} \in P_{m}(t)$ we have

$$
p\left(m n+t^{\prime}\right) \equiv 0 \quad(\bmod l), \quad n \in \mathbb{N} .
$$

Theorem 4.3. Let $Q, a, b, \nu \in \mathbb{N}$ and $t \in\left\{0, \ldots, 2^{a} 3^{b} Q-1\right\}$ be such that $Q, \nu>0$ and $\operatorname{gcd}(Q, 6)=1$. Assume that

$$
p\left(2^{a} 3^{b} Q n+t\right) \equiv 0 \quad(\bmod \nu), \quad n \in \mathbb{N} .
$$

Then

$$
p(Q n+\bar{t}) \equiv 0 \quad(\bmod \nu), \quad n \in \mathbb{N},
$$

where $\bar{t}$ is the minimal non-negative integer such that $t \equiv \bar{t}(\bmod Q)$.

Theorem 4.4. Let $Q, \nu$ be positive integers such that $\operatorname{gcd}(Q, 6 \nu)=1, \nu \neq 1$ and $t \in\{0, \ldots, Q-1\}$. Then there exists an integer $n$ such that $\nu \nmid p(Q n+t)$.

We prove the following lemma.

Lemma 4.5. Let $Q, r, \nu$ be positive integers, $\ell \geq 5$ a prime and $t \in\left\{0, \ldots, Q \ell^{r}-1\right\}$. Let $b$ be the maximal integer such that $\ell^{b} \mid(24 t-1)$. If $r \geq b+1$ and

$$
p\left(Q \ell^{r} n+t\right) \equiv 0 \quad(\bmod \nu), \quad n \in \mathbb{N},
$$

then

$$
p\left(Q \ell^{b+1} n+\bar{t}\right) \equiv 0 \quad(\bmod \nu), \quad n \in \mathbb{N},
$$

where $\bar{t}$ is the minimal non-negative integer such that $t \equiv \bar{t}\left(\bmod Q \ell^{b+1}\right)$.

Before we can prove this result we need the following technical lemma.

Lemma 4.6. Let $Q$ be a positive integer, $v \in \mathbb{Z}$ with $v \neq 0$ and $p \geq 5$ a prime. Let $b$ be maximal such that $p^{b} \mid v$. Then for any $l, r \in \mathbb{Z}$ with $r \geq b+1$ there exists $a_{r, l} \in \mathbb{Z}$ with $\operatorname{gcd}\left(a_{r, l}, 6 Q p\right)=1$ such that

$$
a_{r, l}^{2} v \equiv v+24 Q l p^{b+1} \quad\left(\bmod Q p^{r}\right) .
$$

Proof. Fix $l \in \mathbb{Z}$. By dividing both sides of (9) by $p^{b}$, we may assume that $b=0$ and $p \nmid v$. Then the statement holds for $r=1$ with $a_{r, l}=1$. Next we prove that if the statement is true for $r=R \geq 1$, then it is also true for $r=R+1$. By assumption there exists $a_{R, l}$ such that

$$
a_{R, l}^{2} v \equiv v+24 Q l p \quad\left(\bmod Q p^{R}\right) .
$$

We make the "ansatz" $a_{R+1, l}:=a_{R, l}+24 Q p^{R} x$. Because of (10) there exists $s \in \mathbb{Z}$ satisfying

$$
a_{R, l}^{2} v-v-24 Q l p=Q p^{R} s .
$$

We need to show that there exists $x \in \mathbb{Z}$ such that

$$
\left(a_{R, l}+24 Q p^{R} x\right)^{2} v \equiv v+24 Q l p \quad\left(\bmod Q p^{R+1}\right) .
$$

Together with (11) this reduces to

$$
48 a_{R, l} v x+s \equiv 0 \quad(\bmod p),
$$

which is solvable for $x$ because of $\operatorname{gcd}\left(48 a_{R, l} v, p\right)=1$. Hence the proof is finished by the induction principle. 
Proof of Lemma 4.5. By (8) and Theorem 4.2 we have

$$
p\left(Q \ell^{r} n+t^{\prime}\right) \equiv 0 \quad(\bmod \nu), \quad n \in \mathbb{N}, \quad t^{\prime} \in P_{Q \ell^{r}}(t) .
$$

By Definition 4.1, $t^{\prime} \in P_{Q \ell^{r}}(t)$ iff there exists $a \in \mathbb{Z}$ with $\operatorname{gcd}(a, 6 Q \ell)=1$ such that

$$
a^{2}(24 t-1) \equiv 24 t^{\prime}-1 \quad\left(\bmod Q \ell^{r}\right),
$$

and $t^{\prime} \in\left\{0, \ldots, Q \ell^{r}-1\right\}$. By Lemma 4.6 we obtain that for each $u \in \mathbb{Z}$ there exist $a_{r, u}$ with $\operatorname{gcd}\left(a_{r, u}, 6 Q \ell\right)=1$ such that

$$
a_{r, u}^{2}(24 t-1) \equiv 24\left(t+Q u \ell^{b+1}\right)-1 \quad\left(\bmod Q \ell^{r}\right)
$$

which implies that

$$
\bar{t}+Q u \ell^{b+1} \in P_{Q \ell^{r}}(t) .
$$

Therefore by Theorem 4.2 .

$$
p\left(Q \ell^{r} n+\bar{t}+Q u \ell^{b+1}\right) \equiv 0 \quad(\bmod \nu), \quad n \in \mathbb{N},
$$

for every $u \in\left\{0, \ldots, \ell^{r-b-1}-1\right\}$. Since

$$
Q \ell^{r} n+\bar{t}+Q u \ell^{b+1}=Q \ell^{b+1}\left(\ell^{r-b-1} n+u\right)+\bar{t}
$$

and every non-negative integer $m$ can be written as $m=\ell^{r-b-1} n+u$ for some non-negative integers $n, u$ with $u \in\left\{0, \ldots, \ell^{r-b-1}-1\right\}$, we conclude

$$
p\left(Q \ell^{b+1} m+\bar{t}\right) \equiv 0 \quad(\bmod \nu), \quad m \in \mathbb{N} .
$$

Lemma 4.7. Let $Q$ be a positive integer and $\ell \geq 5$ a prime such that $\operatorname{gcd}(Q, 6 \ell)=1$. Let $\beta \in\{0, \ldots, Q \ell-1\}$. Assume that

$$
p(Q \ell n+\beta) \equiv 0 \quad(\bmod \ell), \quad n \in \mathbb{N} .
$$

Then $\left(\frac{24 \beta-1}{\ell}\right) \neq\left(\frac{-1}{\ell}\right)$.

The proof is based on the following theorem by Deligne and Rapoport.

Theorem 4.8 ([12, VII, Cor. 3.12]). Let $k, N$ be positive integers, $p$ a prime

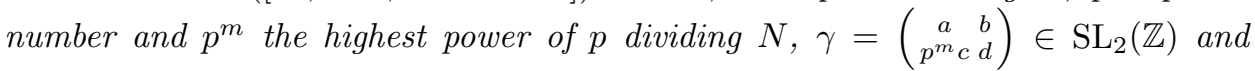
$f \in M_{k}(\Gamma(N))$. Let $\pi$ be a prime ideal in $\mathbb{Z}\left[e^{2 \pi i / N}\right]$ lying above $p$. Assume that the coefficients in the q-expansion of $f$ are in $\mathbb{Z}\left[e^{2 \pi i / N}\right]$. Let $\nu$ be a non-negative integer such that $f \equiv 0\left(\bmod \pi^{\nu}\right)$. Then $\left.f\right|_{k} \gamma \equiv 0\left(\bmod \pi^{\nu}\right)$.

Remark 4.9. For given positive integers $k, N$ and $f \in M_{k}(\Gamma(N))$, with the coefficients of the $q$-expansion of $f$ in $\mathbb{Z}\left[1 / N, e^{2 \pi i / N}\right]$, we obtain by Theorem $4.8[12$, VII, Cor. 3.13] that for $\gamma \in \mathrm{SL}_{2}(\mathbb{Z})$ the coefficients in the $q$-expansion of $\left.f\right|_{k} \gamma$ have the same property. In this case there also exists a power $N^{j}$ of $N$ such that for $\gamma \in \mathrm{SL}_{2}(\mathbb{Z})$ the coefficients in the $q$-expansion of $\left.N^{j} f\right|_{k} \gamma$ are in $\mathbb{Z}\left[e^{2 \pi i / N}\right]$ (see for example [12, VII, Cor 3.11]). Consequently for a given prime $p$ and a prime ideal $\pi$ in $\mathbb{Z}\left[e^{2 \pi i / N}\right]$ lying above $p$, it makes sense to write $\left.f\right|_{k} \gamma \equiv 0\left(\bmod \pi^{\nu}\right)$ if there exists $M \in \mathbb{Z}$ with $M \notin \pi$ such that all the coefficients in the $q$-expansion of $\left.M f\right|_{k} \gamma$ lie in the ideal $\pi^{\nu}$.

We also need some additional simple facts. 
Definition 4.10. For $m$ a positive integer coprime to $6, t \in\{0, \ldots, m-1\}$ and $r \in \mathbb{Z}$, we define

$$
g(m, t, r, \tau):=\frac{1}{m} \sum_{\lambda=0}^{m-1} e^{\frac{2 \pi i \lambda(-24 t-r)}{m}} \eta^{r}\left(\frac{\tau+24 \lambda}{m}\right), \quad \tau \in \mathbb{H} .
$$

The proof of the following lemma is simple and can be found in [22, Lem. 1.12].

Lemma 4.11. Let $m$ be a positive integer coprime to $6, t \in\{0, \ldots, m-1\}$ and $r \in \mathbb{Z}$. Then

$$
g(m, t, r, \tau)=q^{\frac{24 t+r}{24 m}} \sum_{n=0}^{\infty} p_{r}(m n+t) q^{n}, \quad \tau \in \mathbb{H}, \quad\left(q=e^{2 \pi i \tau}\right) .
$$

Proof of Lemma 4.7. Assume that

$$
\left(\frac{24 \beta-1}{\ell}\right)=\left(\frac{-1}{\ell}\right)
$$

Then there exists $a \in \mathbb{Z}$ such that

$$
(24 \beta-1) a^{2} \equiv-1 \quad(\bmod \ell),
$$

which implies together with $\operatorname{gcd}(Q, 6 \ell)=1$ that there exists $\bar{a} \in \mathbb{N}$ with $\operatorname{gcd}(\bar{a}, 6 Q \ell)$ $=1$ such that

$$
\bar{a} \equiv Q a \quad(\bmod \ell)
$$

Let $\bar{\beta} \in\{0, \ldots, Q \ell-1\}$ be uniquely defined by the relation

$$
\bar{a}^{2}(24 \beta-1) \equiv(24 \bar{\beta}-1) \quad(\bmod Q \ell) .
$$

For $k \in \mathbb{Z}$ we have, by Lemma 4.11.

$$
G_{Q \ell, \bar{\beta}}^{(k)}:=\eta^{24 k}\left(q^{\frac{24 \bar{\beta}-1}{24 Q \ell}} \sum_{n=0}^{\infty} p(Q \ell n+\bar{\beta}) q^{n}\right)^{24 Q \ell}=\eta^{24 k}(g(Q \ell, \bar{\beta},-1, \tau))^{24 Q \ell}
$$

where $\eta(\tau):=e^{\pi i \tau / 12} \prod_{n=1}^{\infty}\left(1-e^{2 \pi i n \tau}\right), \tau \in \mathbb{H}$ is the Dedekind eta function and $\eta^{24} \in M_{12}\left(\mathrm{SL}_{2}(\mathbb{Z})\right)$ (see [27, p. 95, Th. 6]). By [22, Lem. 2.10],

$$
\left.(g(Q \ell, \bar{\beta},-1, \tau))^{24 Q \ell}\right|_{-12 Q \ell} \gamma=(g(Q \ell, \bar{\beta},-1, \tau))^{24 Q \ell}
$$

for all $\gamma \in \Gamma_{0}(Q \ell)^{*}$ such that $a^{2} \equiv 1(\bmod Q \ell)$ or equivalently for all $\gamma \in \Gamma_{0}(Q \ell)^{*} \cap$ $\Gamma_{1}^{-}(Q \ell)$. Since, by Lemma 3.1, the set $\Gamma_{0}(Q \ell)^{*} \cap \Gamma_{1}^{-1}(Q \ell)$ generates the group $\Gamma_{1}^{-}(Q \ell) \supseteq \Gamma_{1}(Q \ell)$, it follows that for sufficiently large $k$ we have

$$
G_{Q \ell, \bar{\beta}}^{(k)} \in M_{12(k-Q \ell)}\left(\Gamma_{1}^{-}(Q \ell)\right) \supseteq \Gamma_{1}(Q \ell) .
$$

From now on we fix $k$ to be any integer such that (18) holds.

Let $X, Y$ be integers such that

$$
24^{2} \ell^{2} X+Q Y=1 .
$$

We apply Lemma 5.1 with $t=\bar{\beta}$ and $r=-1$. We then obtain

$$
\begin{aligned}
& e^{-\frac{\pi i Q}{12}} e^{-\frac{48 \pi i X(24 \bar{\beta}-1)}{Q}} g(Q \ell, \bar{\beta},-1, \gamma \tau)(\ell \tau+Q Y)^{1 / 2} \\
= & \frac{1}{Q} \sum_{d \mid Q} d^{-1 / 2} e^{-\frac{\pi i(d-1)}{4}}(-1)^{\frac{Q+1}{2} \frac{d-1}{2}} e^{\frac{2 \pi i\left(24 t_{d}-1\right) d^{2} \tau}{24 Q \ell}} \times \sum_{n=0}^{\infty} e^{\frac{2 \pi i n d^{2} \tau}{Q}} p\left(\ell n+t_{d}\right) T(n, d),
\end{aligned}
$$


where $t_{d}$ is the unique integer satisfying

$$
(24 \bar{\beta}-1) \equiv d^{2}\left(24 t_{d}-1\right) \quad(\bmod \ell), \quad 0 \leq t_{d}<\ell .
$$

Next we observe that $T(n, Q)=1$ for all $n \in \mathbb{N}$ because of $\left(\frac{a}{1}\right)=1$ for $a \in \mathbb{Z}$. Let $m:=Q \ell$ and $q_{m}:=e^{\frac{2 \pi i \tau}{m}}$. Because of (14)-(16) and (21), we have $t_{Q}=0$ and consequently (20) transforms into

$$
\begin{aligned}
& e^{-\frac{\pi i Q}{12}} e^{-\frac{48 \pi i X(24 \bar{\beta}-1)}{Q}} g(Q \ell, \bar{\beta},-1, \gamma \tau)(\ell \tau+Q Y)^{1 / 2} \\
= & \frac{1}{Q} Q^{-1 / 2} e^{-\frac{\pi i(Q-1)}{4}} q_{m}^{-\frac{Q^{2}}{24}} \sum_{n=0}^{\infty} q_{m}^{Q^{2} \ell n} p(\ell n) \\
& +\frac{1}{Q} \sum_{d \mid Q, d \neq Q} d^{-1 / 2} e^{-\frac{\pi i(d-1)}{4}}(-1)^{\frac{Q+1}{2} \frac{d-1}{2}} q_{m}^{\frac{\left(24 t_{d}-1\right) d^{2}}{24}} \sum_{n=0}^{\infty} q_{m}^{n \ell d^{2}} p\left(\ell n+t_{d}\right) T(n, d) \\
= & q_{m}^{-Q^{2} / 24} F\left(q_{m}\right),
\end{aligned}
$$

where

$$
\begin{aligned}
& F\left(q_{m}\right):=\frac{1}{Q} Q^{-1 / 2} e^{-\frac{\pi i(Q-1)}{4}} \sum_{n=0}^{\infty} q_{m}^{Q^{2} \ell n} p(\ell n) \\
& +\frac{1}{Q} \sum_{d \mid Q, d \neq Q} d^{-1 / 2} e^{-\frac{\pi i(d-1)}{4}}(-1)^{\frac{Q+1}{2} \frac{d-1}{2}} q_{m}^{\frac{\left(24 t_{d}-1\right) d^{2}+Q^{2}}{24}} \sum_{n=0}^{\infty} q_{m}^{n \ell d^{2}} p\left(\ell n+t_{d}\right) T(n, d) .
\end{aligned}
$$

We note from (23) that $F\left(q_{m}\right)$ is a Laurent series 1 in powers of $q_{m}$ because of $d^{2}\left(24 t_{d}-1\right)+Q^{2} \equiv 0(\bmod 24)$ because of $Q^{2}, d^{2} \equiv 1(\bmod 24)$. Again from (23) we observe that the coefficients of $F\left(q_{m}\right)$ belong to $\mathbb{Z}\left[1 / Q, e^{2 \pi i / Q}\right]$ because of

$$
T(n, d) \in \mathbb{Z}\left[1 / Q, e^{2 \pi i / Q}\right]
$$

and

$$
d^{-1 / 2} e^{-\frac{\pi i(d-1)}{4}} \in \mathbb{Z}\left[1 / Q, e^{2 \pi i / Q}\right],
$$

for $d \mid Q$. The relation (24) is seen directly from the definition of $T(n, d)$. To prove (25) we use the relation $d^{1 / 2} e^{\frac{\pi i(d-1)}{4}}= \pm \epsilon(d) d^{1 / 2}$, where

$$
\epsilon(d):=\left\{\begin{array}{lll}
1, & \text { if } d \equiv 1 \quad(\bmod 4), \\
i, & \text { if } d \equiv 3 \quad(\bmod 4) .
\end{array}\right.
$$

By [17, p. 87] we have $\epsilon(d) d^{1 / 2}=\sum_{\lambda=0}^{d-1} e^{2 \pi i \lambda^{2} / d}$. This implies that

$$
d^{-1 / 2} e^{-\frac{\pi i(d-1)}{4}}=d^{-1}(-1)^{\frac{d-1}{2}} \sum_{\lambda=0}^{d-1} e^{2 \pi i \lambda^{2} / d} \in \mathbb{Z}\left[1 / Q, e^{2 \pi i / Q}\right] .
$$

Furthermore, if $\pi$ is a prime ideal in $\mathbb{Z}\left[e^{\frac{2 \pi i}{\ell Q}}\right]$ lying above $\ell$, then it makes sense to reduce the coefficients of $F\left(q_{m}\right)$ modulo $\pi$ because the coefficients of $F\left(q_{m}\right)$ are in $\mathbb{Z}\left[1 / Q, e^{2 \pi i / Q}\right]$ and $Q$ is invertible modulo $\pi$. We observe that

$$
F\left(q_{m}\right) \not \equiv 0 \quad(\bmod \pi)
$$

\footnotetext{
${ }^{1}$ In fact $F\left(q_{m}\right)$ is a Laurent series in powers of $q_{m}^{\ell}$ because of $d^{2}\left(24 t_{d}-1\right)+Q^{2} \equiv 0(\bmod \ell)$ because of (14) -16 and 21.
} 
because by (22) the order of $F\left(q_{m}\right)$ is 0 and the coefficient of the constant term is equal to $\frac{1}{Q^{3 / 2}} e^{-\frac{\pi i(Q-1)}{4}} \not \equiv 0(\bmod \pi)$.

By (22), Definition 4.10 and Lemma 4.11 we have

$$
\left.G_{Q \ell, \bar{\beta}}^{(k)}\right|_{\kappa}\left(\begin{array}{cc}
1 & -24^{2} \ell X \\
\ell & Q Y
\end{array}\right)=\eta^{24 k} q^{-Q^{2}} F^{24 Q \ell}\left(q_{m}\right),
$$

where $\kappa:=12(k-Q \ell)$. Then (26) implies

$$
\left.G_{Q \ell, \bar{\beta}}^{(k)}\right|_{\kappa}\left(\begin{array}{cc}
1-24^{2} \ell X \\
\ell & Q Y
\end{array}\right) \not \equiv 0 \quad(\bmod \pi)
$$

and by Theorem 4.8 .

$$
G_{Q \ell, \bar{\beta}}^{(k)} \not \equiv 0 \quad(\bmod \pi),
$$

and consequently $p(Q \ell n+\bar{\beta}) \not \equiv 0(\bmod \ell)$ for some $n \in \mathbb{N}$ and since $\beta \in P_{Q \ell}(\bar{\beta})$ because of (16) and Definition 4.1, we obtain by Theorem 4.2 that $p\left(Q \ell n^{\prime}+\beta\right) \not \equiv 0$ $(\bmod \ell)$ for some $n^{\prime} \in \mathbb{N}$ which is a contradiction to our assumption (13).

\section{A MOdUlar SUbstitution FORMUla}

Lemma 5.1. Let $\ell \geq 5$ be a prime and $Q$ a positive integer coprime to $6, t \in$ $\{0, \ldots, m-1\}, r \in \mathbb{Z}$ and $X, Y \in \mathbb{Z}$ such that

$$
24^{2} \ell^{2} X+Q Y=1 \text {. }
$$

Let $\gamma:=\left(\begin{array}{cc}1 & -24^{2} \ell X \\ \ell & Q Y\end{array}\right) \in \mathrm{SL}_{2}(\mathbb{Z})$. For any integers $s$ and $d \mid Q$ such that $\operatorname{gcd}(s, Q / d)=$ 1 , let $\iota_{s, d}$ be any integer satisfying $s \cdot \iota_{s, d} \equiv 1(\bmod Q / d)$. Then

$$
\begin{aligned}
& e^{\frac{\pi i r Q}{12}} e^{-\frac{48 \pi i X(24 t+r)}{Q}} g(Q \ell, t, r, \gamma \tau)(\ell \tau+Q Y)^{-r / 2} \\
= & \frac{1}{Q} \sum_{d \mid Q} e^{\frac{\pi i r(d-1)}{4}} d^{r / 2}(-1)^{r \frac{Q+1}{2} \frac{d-1}{2}} e^{\frac{2 \pi i\left(24 t_{d}+r\right) d^{2} \tau}{24 Q \ell}} \sum_{n=0}^{\infty} e^{\frac{2 \pi i n d^{2} \tau}{Q}} p_{r}\left(\ell n+t_{d}\right) T(n, d),
\end{aligned}
$$

where $t_{d} \in\{0, \ldots, \ell-1\}$ is the unique integer satisfying

$$
24 t+r \equiv d^{2}\left(24 t_{d}+r\right) \quad(\bmod \ell)
$$

and

$$
T(n, d):=\sum_{\substack{0 \leq s<Q / d \\ \operatorname{gcd}(s, Q / d)=1}}\left(\frac{24 \ell s}{Q / d}\right)^{r} e^{-\frac{48 \pi i X}{Q / d}\left\{\iota_{s, d}\left(24\left(\ell n+t_{d}\right)+r\right)+s(24 t+r)\right\}} .
$$

Proof. Note that for any $\lambda \in\{0, \ldots, \ell Q-1\}$ we have $\lambda \equiv-24 \ell X+s d+l Q(\bmod \ell Q)$ for some unique $(d, s, l) \in S$, where

$$
S:=\{(d, s, l): d \mid Q, s \in\{0, \ldots, Q / d\}, \operatorname{gcd}(s, Q / d)=1, l \in\{0, \ldots, \ell-1\}\} .
$$

This is proven by observing that $|S|:=\ell \sum_{d \mid Q} \varphi(Q / d)=\ell Q$ and by proving that if $\lambda_{1}=-24 \ell X+s_{1} d_{1}+l_{1} Q$ and $\lambda_{2}=-24 \ell X+s_{2} d_{2}+l_{2} Q$ satisfy $\lambda_{1} \equiv \lambda_{2}(\bmod \ell Q)$ 
for some $\left(d_{1}, s_{1}, l_{1}\right),\left(d_{2}, s_{2}, l_{2}\right) \in S$, then $\left(d_{1}, s_{1}, l_{1}\right)=\left(d_{2}, s_{2}, l_{2}\right)$. By Definition 4.10 we obtain

(30)

$$
\begin{aligned}
& g(Q \ell, t, r, \gamma \tau) \\
= & \frac{1}{Q \ell} \sum_{d \mid Q} \sum_{\substack{0 \leq s<\frac{Q}{d}-1 \\
\operatorname{gcd}(s, m Q / d)=1}} \sum_{l=0}^{m_{C}-1} e^{\frac{2 \pi i(-24 \ell X+s d+l Q)(-24 t-r)}{Q \ell}} \eta^{r}\left(\frac{\gamma \tau+24(-24 \ell X+s d+l Q)}{Q \ell}\right) .
\end{aligned}
$$

We fix $(d, l, s) \in S$ and define

$$
\lambda:=-24 \ell X+s d+l Q .
$$

One verifies that

$$
\frac{\gamma \tau+24 \lambda}{m}=M_{\lambda} \frac{d \tau+(-24 \ell X+\lambda Q Y) 24^{2} x_{\lambda}}{Q \ell / d},
$$

where $M_{\lambda}:=\left(\begin{array}{cc}\frac{1+24 \lambda \ell}{d} & 24 \ell y_{\lambda}(-24 \ell X+\lambda Q Y) \\ \frac{\ell^{2} Q}{d} & \ell^{2} Q^{2} Y y_{\lambda}+24 x_{\lambda}\end{array}\right)$ and $x_{\lambda}, y_{\lambda}$ are integers such that

$$
24(24 \lambda \ell+1) x_{\lambda}+\ell^{2} Q y_{\lambda}=d .
$$

Knopp [16, p. 51] proved that for each $\xi=\left(\begin{array}{ll}a & b \\ c & d\end{array}\right) \in \mathrm{SL}_{2}(\mathbb{Z})$ with $c$ odd we have

$$
\eta(\xi \tau)=(c \tau+d)^{1 / 2} v_{\eta}(\xi) \eta(\tau), \quad \tau \in \mathbb{H},
$$

where

$$
v_{\eta}(\xi):=\left(\frac{d}{|c|}\right) e^{\frac{\pi i}{12}\left(c(a+d)-b d\left(c^{2}-1\right)-3 c\right)} .
$$

By (32) and (34) we obtain

$$
\eta\left(\frac{\gamma \tau+24 \lambda}{Q \ell}\right)=(d(\ell \tau+Q Y))^{1 / 2} v_{\eta}\left(M_{\lambda}\right) \eta\left(\frac{d \tau+24^{2} x_{\lambda}(-24 \ell X+\lambda Q Y)}{Q \ell / d}\right) .
$$

We have $v_{\eta}\left(M_{\lambda}\right)=\left(\frac{24 x_{\lambda}}{Q / d}\right) e^{\frac{\pi i Q}{12}(2-3 d)}$ because of $M_{\lambda} \equiv\left(\begin{array}{cc}d & 0 \\ Q d & d\end{array}\right) \quad(\bmod 24)$ because of $d^{2} \equiv 1(\bmod 24)$ and because of $y_{\lambda} \equiv Q d(\bmod 24)$ by (33). By (31) and (33) we obtain

$$
24 x_{\lambda} \cdot 24 \ell s \equiv 1 \quad(\bmod Q / d)
$$

which implies $\left(\frac{24 x_{\lambda}}{Q / d}\right)=\left(\frac{24 \ell s}{Q / d}\right)$ by using standard properties of the Jacobi symbol. Consequently, we obtain

$$
v_{\eta}\left(M_{\lambda}\right)=\left(\frac{24 \ell s}{Q / d}\right) e^{\frac{\pi i Q}{12}(2-3 d)}=\left(\frac{24 \ell s}{Q / d}\right) e^{\frac{\pi i(d-1)}{4}} e^{-\frac{\pi i Q}{12}}(-1)^{\frac{Q+1}{2} \frac{d-1}{2}} .
$$

By (36) and (38) we obtain

$$
\begin{aligned}
& e^{\frac{\pi i Q}{12}}(\ell \tau+Q Y)^{-1 / 2} \eta\left(\frac{\gamma \tau+24 \lambda}{Q \ell}\right) \\
= & d^{1 / 2} e^{\frac{\pi i(d-1)}{4}}\left(\frac{24 \ell s}{Q / d}\right)(-1)^{\frac{Q+1}{2} \frac{d-1}{2}} \eta\left(\frac{d \tau+24^{2} x_{\lambda}(-24 \ell X+\lambda Q Y)}{Q \ell / d}\right) .
\end{aligned}
$$

Next we obtain an expression for $H:=24 x_{\lambda}(-24 \ell X+\lambda Q Y)$ modulo $\ell Q / d$. 
Multiplying both sides of (37) by $\ell X \iota_{s, d}$ we obtain $x_{\lambda} \equiv \ell X \iota_{s, d}(\bmod Q / d)$ because of $s \iota_{s, d} \equiv 1(\bmod Q / d)$ and $24^{2} \ell^{2} X \equiv 1(\bmod Q / d)$ because of (27)). This implies that

$$
H \equiv-24^{2} \ell X \cdot \ell X \iota_{s, d} \equiv-X i_{s, d} \quad(\bmod Q / d) .
$$

By (33) $)$ we obtain $x_{\lambda} \equiv d / 24(\bmod \ell)$. This implies together with (31) that

$$
H \equiv 24(s d+l Q) Q Y \cdot d / 24 \equiv s d^{2}+l Q d \quad(\bmod \ell)
$$

because of $Q Y \equiv 1(\bmod \ell)$ because of (27).

Because of (41) there exists an integer $v$ such that $H=s d^{2}+l Q d+v \ell$ which implies, together with (40) and $24^{2} \ell^{2} X \equiv 1$ by (27), that

$$
v \equiv 24^{2} X \ell\left(-X \iota_{s, d}-s d^{2}-l Q d\right) \text {. }
$$

Consequently,

$$
\begin{aligned}
H & \equiv s d^{2}+l Q d+24^{2} \ell^{2} X\left(-X \iota_{s, d}-s d^{2}-l Q d\right)=Q Y\left(s d^{2}+l Q d\right)-24^{2} \ell^{2} X^{2} \iota_{s, d} \\
& \equiv s d^{2} Y Q+l d Q-24^{2} \ell^{2} X^{2} \iota_{s, d} \quad(\bmod \ell Q / d) .
\end{aligned}
$$

Next note that if $v_{1}$ and $v_{2}$ are integers such that $v_{2}=v_{1}+t(24 Q \ell / d)$ for some integer $t$, then $\eta\left(\frac{d \tau+v_{2}}{\ell Q / d}\right)=\eta\left(\frac{d \tau+v_{1}}{\ell Q / d}+24 t\right)=\eta\left(\frac{d \tau+v_{1}}{Q \ell / d}\right)$, because of $\eta(\tau+24)=$ $\eta(\tau)$. Using this fact with $v_{1}=24 H$ and $v_{2}=24\left(s d^{2} Y Q+l d Q-24^{2} \ell^{2} X^{2} \iota_{s, d}\right)$ on (39), we obtain

$$
\begin{aligned}
& e^{\frac{\pi i Q}{12}}(\ell \tau+Q Y)^{-1 / 2} \eta\left(\frac{\gamma \tau+24 \lambda}{Q \ell}\right) \\
= & d^{1 / 2} e^{\frac{\pi i(d-1)}{4}}\left(\frac{24 \ell s}{Q / d}\right)(-1)^{\frac{Q+1}{2} \frac{d-1}{2}} \eta\left(\frac{\tau^{\prime}(s, d)+24 d^{2} l}{\ell}\right),
\end{aligned}
$$

where

$$
\tau^{\prime}(s, d):=\frac{d \tau+24\left(s d^{2} Y Q-24^{2} \ell^{2} X^{2} \iota_{s, d}\right)}{Q / d}
$$

By (42) and (30)

$$
\begin{aligned}
& e^{\frac{\pi i r Q}{12}}(\ell \tau+Y Q)^{-r / 2} g(\ell Q, t, r, \gamma \tau) \\
= & \frac{1}{\ell Q} \sum_{d \mid Q} \sum_{\substack{0 \leq s<\frac{Q}{d}-1 \\
\operatorname{gcd}(s, Q / d)=1}}^{\ell-1} \sum_{l=0} e^{\frac{2 \pi i(-24 \ell X+s d+l Q)(-24 t-r)}{\ell Q}} \\
& \times d^{r / 2} e^{\frac{\pi i r(d-1)}{4}}(-1)^{r \frac{Q+1}{2} \frac{d-1}{2}}\left(\frac{24 \ell s}{Q / d}\right)^{r} \eta^{r}\left(\frac{\tau^{\prime}(s, d)+24 d^{2} l}{\ell}\right) \\
= & e^{\frac{48 \pi i X(24 t+r)}{Q}} \frac{1}{\ell Q} \sum_{d \mid Q} d^{r / 2} e^{\frac{\pi i r(d-1)}{4}} \sum_{\substack{0 \leq s<\frac{m_{0}}{d}-1 \\
\operatorname{gcd}(s, Q / d)=1}} e^{\frac{2 \pi i s(-24 t-r)}{\ell Q / d}} \\
& \times \sum_{l=0}^{\ell-1} e^{\frac{2 \pi i l(-24 t-r)}{\ell}} e^{\frac{\pi i r(d-1)}{4}}(-1)^{r \frac{Q+1}{2} \frac{d-1}{2}}\left(\frac{24 \ell s}{Q / d}\right)^{r} \eta^{r}\left(\frac{\tau^{\prime}(s, d)+24 d^{2} l}{\ell}\right) .
\end{aligned}
$$

Summing in the last sum over any set of modulo $\ell$ representatives does not change the value of the sum. In particular, we make the substitution $l=(Y Q / d)^{2} l^{\prime}$ and 
observe that $d^{2}(Y Q / d)^{2} \equiv 1(\bmod \ell)$ because of (27). Thus we obtain together with Definition 4.10 and (29),

$$
\begin{aligned}
& e^{\frac{\pi i r Q}{12}} e^{-\frac{48 \pi i X(24 t+r)}{Q}} g(m, t, r, \gamma \tau)(\ell \tau+Y Q)^{-r / 2} \\
= & \frac{1}{Q} \sum_{d \mid Q} d^{r / 2} e^{\frac{\pi i r(d-1)}{4}}(-1)^{r \frac{Q+1}{2} \frac{d-1}{2}} \sum_{\substack{0 \leq s<Q / d \\
\operatorname{gcd}(s, Q / d)}}\left(\frac{24 \ell s}{Q / d}\right)^{r} e^{\frac{2 \pi i(-24 t-r) s}{\ell Q / d}} g\left(\ell, t_{d}, r, \tau^{\prime}(s, d)\right) .
\end{aligned}
$$

By Lemma 4.11.

$$
\begin{aligned}
& \sum_{\substack{0 \leq s<Q / d \\
\operatorname{gcd}(s, Q / d)}}\left(\frac{s}{Q / d}\right)^{r} e^{\frac{2 \pi i(-24 t-r) s}{\ell Q / d}} g\left(\ell, t_{d}, r, \tau^{\prime}(s, d)\right) \\
& =\sum_{\substack{0 \leq s<Q / d \\
\operatorname{gcd}(s, Q / d)}}\left(\frac{s}{Q / d}\right)^{r} e^{\frac{2 \pi i(-24 t-r) s}{\ell Q / d}} e^{\frac{2 \pi i\left(24 t_{d}+r\right) \tau^{\prime}(s, d)}{24 \ell}} \sum_{n=0}^{\infty} p_{r}\left(\ell n+t_{d}\right) e^{2 \pi i n \tau^{\prime}(s, d)} \\
& =\sum_{\substack{0 \leq s<Q / d \\
\operatorname{gcd}(s, Q / d)}}\left(\frac{s}{Q / d}\right)^{r} e^{\frac{2 \pi i(-24 t-r) s}{\ell Q / d}} e^{\frac{2 \pi i\left(24 t_{d}+r\right)\left\{\frac{d \tau+24\left(s d^{2} Y Q-24^{2} \ell^{2} X^{2} \iota_{s, d}\right)}{Q / d}\right\}}{24 \ell}} \\
& \times \sum_{n=0}^{\infty} p_{r}\left(\ell n+t_{d}\right) e^{2 \pi i n\left\{\frac{d \tau+24\left(s d^{2} Y Q-24^{2} \ell^{2} X^{2} \iota_{s, d}\right)}{Q / d}\right\}} \\
& =e^{\frac{2 \pi i d^{2}\left(24 t_{d}+r\right) \tau}{24 \ell Q}} \sum_{n=0}^{\infty} p_{r}\left(\ell n+t_{d}\right) e^{\frac{2 \pi i n d^{2} \tau}{Q}} \\
& \times \sum_{\substack{0 \leq s<Q / d \\
\operatorname{gcd}(s, Q / d)}}\left(\frac{s}{Q / d}\right)^{r} e^{-\frac{2 \pi i}{\ell Q / d}\left\{24^{2} \ell^{2} X^{2} \iota_{s, d}\left(\left(24 t_{d}+r\right)+24 \ell n\right)+s\left(24 t+r-\left(24 t_{d}+r\right) d^{2} Q Y\right)\right\}} \\
& =e^{\frac{2 \pi i d^{2}\left(24 t_{d}+r\right) \tau}{24 \ell Q}} \sum_{n=0}^{\infty} p_{r}\left(\ell n+t_{d}\right) e^{\frac{2 \pi i n d^{2} \tau}{Q}} \\
& \times \underbrace{\sum_{\substack{0 \leq s<Q / d \\
\operatorname{gcd}(s, Q / d)}}\left(\frac{s}{Q / d}\right)^{r} e^{-\frac{48 \pi i \ell X}{m / d}\left\{24 \ell X \iota_{s, d}\left(24\left(n \ell+t_{d}\right)+r\right)+24 \ell s(24 t+r)\right\}},}_{=T(n, d)}
\end{aligned}
$$

by first substituting $d^{2} Q Y\left(24 t_{d}+r\right) \equiv Q Y(24 t+r)(\bmod \ell Q)$ which follows from (29) and next substituting $1-Q Y=24^{2} \ell^{2} X$ because of (27). Next we exploit the identity,

$$
\begin{aligned}
T(n, d) & =\sum_{\substack{0 \leq s<Q / d \\
\operatorname{gcd}(s, Q / d)=1}}\left(\frac{s}{Q / d}\right)^{r} e^{-\frac{48 \pi i \ell X}{m / d}\left\{24 \ell x \iota_{s, d}\left(24\left(n \ell+t_{d}\right)+r\right)+24 \ell s(24 t+r)\right\}} \\
& =\sum_{\substack{0 \leq s<Q / d \\
\operatorname{gcd}(s, Q / d)=1}}\left(\frac{24 \ell s}{Q / d}\right)^{r} e^{-\frac{48 \pi i X}{Q / d}\left\{\iota_{s, d}\left(24\left(n \ell+t_{d}\right)+r\right)+s(24 t+r)\right\}}
\end{aligned}
$$

because $s \mapsto 24 \ell X s$ is a bijection modulo $Q / d$ together with $24^{2} \ell^{2} x \equiv 1(\bmod Q / d)$.

Finally substituting in (44) we obtain (28). 


\section{ACKNOWLEDGEMENT}

The author thanks his advisor Peter Paule. His suggestions improved the quality of this manuscript. The idea on how to solve the problem treated here is due to the ideas and questions that arose in our first paper 22 in which Peter Paule was very influential through his algorithmic view of mathematical problems. The author also thanks Scott Ahlgren and Ken Ono for posing the problem. Finally, the autor thanks the anonymous referee for suggestions that improved the content of this paper.

\section{REFERENCES}

1. S. Ahlgren, Distribution of the Partition Function Modulo Composite Integers M, Mathematische Annalen 318 (2000), no. 4, 795-803. MR1802511 (2001j:11099)

2. S. Ahlgren and M. Boylan, Arithmetic Properties of the Partition Function, Inventiones Mathematicae 153 (2003), no. 3, 487-502. MR2000466 (2004e:11115)

3. S. Ahlgren and K. Ono, Congruence Properties for the Partition Function, Proceedings of the National Academy of Science 98 (2001), no. 23, 12882-12884. MR.1862931(2002k:11187)

4. __ Congruences and Conjectures for the Partition Function, q-Series with applications to combinatorics, number theory, and physics (Urbana, IL, 2000), Contemporary Mathematics, vol. 291, AMS, 2001, pp. 1-10. MR1874518 (2002j:11120)

5. G. Andrews and F. Garvan, Dyson's Crank of a Partition, Bulletin of the American Mathematical Society 18 (1988), no. 2, 167-171. MR929094(89b:11079)

6. A. O. L. Atkin, Proof of a Conjecture of Ramanujan, Glasgow Mathematical Journal 8 (1967), 14-32. MR0205958 (34:5783)

7. __ Multiplicative Congruence Properties and Densitiy Problems for $p(n)$, Proceedings of London Mathematical Society 18 (1968), 563-576. MR0227105 (37:2690)

8. A. O. L. Atkin and J. N. O'Brien, Some Properties of $p(n)$ and $c(n)$ Modulo Powers of 13, Transactions of the American Mathematical Society 126 (1967), 442-459. MR.0214540 $(35: 5390)$

9. A. O. L. Atkin and P. Swinnerton-Dyer, Some Properties of Partitions, Proceedings of Londons Mathematical Society 4 (1954), 84-106. MR0060535(15:685d)

10. Modular Forms on Noncongruence Subgroups, Combinatorics (Proc. Sympos. Pure., Math., Vol. XIX, Univ. California, Los Angeles, Calif., 1968), American Mathematical Society, 1971, pp. 1-25. MR0337781 (49:2550)

11. B. C. Berndt and K. Ono, Ramanujan's Unpublished Manuscript on the Partition and Tau Functions with Proofs and Commentary, Séminaire Lotharingien de Combinatoire 42 (1999), Art. B42c, 63pp. (electronic). MR 1701582 (2000i:01027)

12. P. Deligne and M. Rapoport, Les Schémas de Modules de Courbes Elliptiques, Modular Functions of one Variable, II (Proc. Internat. Summer School, Univ. Antwerp, Antwerp 1972), Springer-Verlag Berlin, 1973, pp. 143-316. Lecture Notes in Math., Vol. 349. MR0337993 $(49: 2762)$

13. F. J. Dyson, Some Guesses in the Theory of Partitions, Eureka 8 (1944), 11-15.

14. D. Kim, F. Garvan and D. Stanton, Cranks and t-Cores, Inventiones Mathematicae 101 (1990), no. 1, 1-17. MR.1055707 (91h:11106)

15. I. Kiming and J. B. Olsson, Congruences like Ramanujan's for Powers of the Partition Function, Archiv der Mathematik 59 (1992), no. 4, 348-360. MR1179461 (93g:11105)

16. M. I. Knopp, Modular Functions in Analytic Number Theory, American Mathematical Society, 1993.

17. S. Lang, Algebraic Number Theory, Addison-Wesley Publishing Company, Inc., 1970. MR0282947(44:181)

18. R. Lewis, The Components of Modular Forms, J. London Math. Soc. 52 (1995), 245-254. MR.1356140 (96g:11126)

19. M. Newman, Note on Partitions Modulo 5, Mathematics of Computation 21 (1967), 481-482. $\operatorname{MR} 0227127(37: 2712)$

20. K. Ono, Distribution of the Partition Function Modulo m, Annals of Mathematics 151 (2000), no. 1, 293-307. MR.1745012(2000k:11115) 
21. S. Radu, A Proof of Subbarao's Conjecture, to appear in Crelle.

22. _ An Algorithmic Approach to Ramanujan's Congruences, Ramanujan Journal 20 (2009), 215-251. MR2558703 (2010j:11151)

23. S. Ramanujan, On Certain Arithmetical Functions, Transactions of Cambridge Philosophical Society 22 (1916), no. 9, 159-184.

24. Some Properties of $p(n)$ the Number of Partitions of $n$, Proceedings of Cambridge Philosophical Society 19 (1919), 207-210.

25. Congruence Properties of Partitions, Proceedings of London Mathematical Society 18 (1920), no. 2, xix.

26. Congruence Properties of Partitions, Mathematische Zeitschrift 9 (1921), no. 1-2, 147-153. MR1544457

27. J. P. Serre, A Course in Arithmetic, Springer, 1973. MR0344216 (49:8956)

28. __ Formes Modulaires et Functions zêta p-adiques, Modular functions of one variable, III (Proc. Internat. Summer School, Univ. Antwerp, 1972), Springer, 1973, pp. 191-268. Lecture Notes in Math., Vol. 350. MR0404145(53:7949a)

29. H. P. F. Swinnerton-Dyer, On $\ell$-adic Representations and Congruences for Coefficients of Modular Forms, Modular functions of one variable, III (Proc. Internat. Summer School, Univ. Antwerp, 1972), Springer, 1973, pp. 1-55. Lecture Notes in Math., Vol. 350. MR0406931 $(53: 10717 \mathrm{a})$

Research Institute for Symbolic Computation (RISC), Johannes Kepler University, A-4040 Linz, Austria 